\title{
T-Duality and Mixed Branes
}

\author{
Davoud Kamani \\ Institute for Studies in Theoretical Physics and Mathematics (IPM) \\ P.O.Box: 19395-5531, Tehran, Iran \\ e-mail: kamani@theory.ipm.ac.ir
}

\begin{abstract}
In this article the action of T-duality on a mixed brane is studied in the boundary state formalism. We also obtain a two dimensional mixed brane with non-zero electric and magnetic fields, from a $\mathrm{D}_{1}$-brane.
\end{abstract}

PACS:11.25.-w; 11.25.Mj; 11.30.pb 


\section{Introduction}

It is well known that the T-duality in string theory is the target space duality, and generalizes the $R \rightarrow \alpha^{\prime} / R$ duality in compactified string theory [1, 2, 3, 4, 5]. In fact, T-duality transformation is an exact symmetry of closed string theory [4], and it can be used to relate problems that might at first sight seem quite different.

In type II superstring theories, the action of T-duality on $k$-directions (compactified on tori) of a $\mathrm{D}_{p}$-brane produces a $\mathrm{D}_{p-k}$-brane, and on $k$-directions, perpendicular to a $\mathrm{D}_{p^{-}}$ brane, produces a $\mathrm{D}_{p+k}$-brane. Therefore for odd " $k$ ", type IIA theory changes to the type IIB theory and vice-versa [2, 6, 7]. In other words, the IIA theory compactified on a circle of radius $R$ is equivalent to the IIB theory compactified on a circle of radius $\alpha^{\prime} / R$.

In this note we are interested in exploring what happens to a brane with internal background field, under the action of the T-duality within the boundary state formalism. The boundary conditions corresponding to the closed string, emitted from this brane, are combinations of Dirichlet and Neumann boundary conditions, therefore we name it, "mixed brane", or $\mathrm{m}_{p}$-brane [2, 8, 9, 10].

In section 2, we apply T-duality on the boundary state equations, corresponding to a mixed brane. We shall see that the action of T-duality on two directions of certain mixed branes does not change their dimensions, unlike D-branes. It instead changes the background field strength. In section 3 , with the appropriate T-dualities we obtain a two dimensional mixed brane with all field components, from a $\mathrm{D}_{1}$-brane. The process can be generalized to obtain higher dimensional branes with internal back-ground fields.

\section{T-duality on mixed boundary conditions}

The boundary conditions for a closed string emitted from a $\mathrm{m}_{p}$-brane are [2, 8, 9],

$$
\begin{gathered}
\left(\partial_{\tau} X^{\alpha}+\mathcal{F}_{\beta}^{\alpha} \partial_{\sigma} X^{\beta}\right)_{\tau_{0}}|B\rangle=0, \\
\left(\partial_{\sigma} X^{i}\right)_{\tau_{0}}|B\rangle=0
\end{gathered}
$$

for the bosonic part, and

$$
\begin{gathered}
\left(\left(\psi^{\alpha}-i \eta \tilde{\psi}^{\alpha}\right)-\mathcal{F}_{\beta}^{\alpha}\left(\psi^{\beta}+i \eta \tilde{\psi}^{\beta}\right)\right)_{\tau_{0}}|B\rangle=0, \\
\left(\psi^{i}+i \eta \tilde{\psi}^{i}\right)_{\tau_{0}}|B\rangle=0
\end{gathered}
$$


for the fermionic part, where $\tau_{0}$ is the $\tau$ variable on the boundary of the closed string world sheet and $\eta= \pm 1$. The indices $\alpha$ and $\beta$ show the brane directions and $i$ and $j$ show the directions perpendicular to it. Total field strength is

$$
\mathcal{F}_{\alpha \beta}=\partial_{[\alpha} A_{\beta]}-B_{\alpha \beta}
$$

where $A_{\alpha}$ is a $U(1)$ gauge field which lives on the brane, and $B_{\mu \nu}$ is the antisymmetric tensor of the $\mathrm{NS} \otimes \mathrm{NS}$ sector of the type II superstring theories as back-ground, in the bulk of space-time.

Solution of the equations (1)-(4) gives the boundary state $|B\rangle$, which describes the mixed brane [8, 9].

Now consider T-duality in type II superstring theories. The closed string mode expansion is

$$
\begin{gathered}
X^{\mu}(\sigma, \tau)=X_{L}^{\mu}(\tau+\sigma)+X_{R}^{\mu}(\tau-\sigma) \\
X_{L}^{\mu}(\tau+\sigma)=x_{L}^{\mu}+2 \alpha^{\prime} p_{L}^{\mu}(\tau+\sigma)+\frac{i}{2} \sqrt{2 \alpha^{\prime}} \sum_{n \neq 0} \frac{1}{n} \tilde{\alpha}_{n}^{\mu} e^{-2 i n(\tau+\sigma)}, \\
X_{R}^{\mu}(\tau-\sigma)=x_{R}^{\mu}+2 \alpha^{\prime} p_{R}^{\mu}(\tau-\sigma)+\frac{i}{2} \sqrt{2 \alpha^{\prime}} \sum_{n \neq 0} \frac{1}{n} \alpha_{n}^{\mu} e^{-2 i n(\tau-\sigma)} .
\end{gathered}
$$

For non-compact direction $X^{\mu}$, we have $p_{L}^{\mu}=p_{R}^{\mu}=\frac{1}{2} p^{\mu}$, since $X^{\mu}$ is single valued at $\sigma$ and $\sigma+\pi$. If the direction $X^{\mu}$ is compact on a circle of radius $R$, the left and the right components of the momentum are

$$
\begin{aligned}
& p_{L}^{\mu}=\frac{1}{2 \sqrt{\alpha^{\prime}}}\left(\frac{\sqrt{\alpha^{\prime}}}{R} M+\frac{R}{\sqrt{\alpha^{\prime}}} N\right), \\
& p_{R}^{\mu}=\frac{1}{2 \sqrt{\alpha^{\prime}}}\left(\frac{\sqrt{\alpha^{\prime}}}{R} M-\frac{R}{\sqrt{\alpha^{\prime}}} N\right),
\end{aligned}
$$

where the integers $N$ and $M$ are winding and momentum numbers of closed string respectively. Under the transformations

$$
\frac{R}{\sqrt{\alpha^{\prime}}} \leftrightarrow \frac{\sqrt{\alpha^{\prime}}}{R} \quad, \quad M \leftrightarrow N,
$$

the mass spectrum and interactions of the string theory are invariant. The T-duality transformation on the compact direction $X^{\mu}$ can be written

$$
T_{\mu}: \quad X^{\mu}(\sigma, \tau) \rightarrow X^{\prime \mu}(\sigma, \tau)=X_{L}^{\mu}-X_{R}^{\mu}
$$


which is a space-time parity transformation acting only on the right moving degree of freedom. According to the (6)-(8), this gives transformations of (11) and

$$
\partial_{\tau} X^{\mu} \leftrightarrow \partial_{\sigma} X^{\mu}
$$

i.e. under the T-duality, the Dirichlet and Neumann boundary conditions get exchanged. Worldsheet supersymmetry requires the following transformations for the worldsheet fermions

$$
T_{\mu}: \quad \psi^{\mu} \rightarrow-\psi^{\mu} \quad, \quad \tilde{\psi}^{\mu} \rightarrow \tilde{\psi}^{\mu}
$$

these transformations cause the space-time metric $G_{\mu \nu}$ and field strength $\mathcal{F}_{\mu \nu}$ to transform under the T-duality, which can be obtained by observing their action on the NS $\otimes$ NS sector oscillators

$$
\begin{aligned}
& \left|G^{\mu \nu}\right\rangle=\left(b_{-1 / 2}^{\mu} \tilde{b}_{-1 / 2}^{\nu}+b_{-1 / 2}^{\nu} \tilde{b}_{-1 / 2}^{\mu}\right)|0\rangle, \\
& \left|B^{\mu \nu}\right\rangle=\left(b_{-1 / 2}^{\mu} \tilde{b}_{-1 / 2}^{\nu}-b_{-1 / 2}^{\nu} \tilde{b}_{-1 / 2}^{\mu}\right)|0\rangle .
\end{aligned}
$$

Under the transformations (14), we have $b_{-1 / 2}^{\mu} \rightarrow-b_{-1 / 2}^{\mu}$ and $\tilde{b}_{-1 / 2}^{\mu} \rightarrow \tilde{b}_{-1 / 2}^{\mu}$, therefore,

$$
T_{\mu}:\left\{\begin{array}{l}
G^{\mu \nu} \leftrightarrow-B^{\mu \nu}, \quad \nu \neq \mu \\
G^{\mu \mu} \rightarrow-G^{\mu \mu} .
\end{array}\right.
$$

We shall also observe from consistency that the transformations between $\eta_{\mu \nu}$ and $\mathcal{F}_{\mu \nu}$ have similar form.

Now consider brane direction $X^{\alpha_{c}}$ which is assumed to be compact, therefore $\mathrm{T}_{\alpha_{c}}$-duality acts on the boundary conditions (1)-(4) as

$$
\begin{aligned}
& \left\{\begin{array}{l}
\left(\partial_{\tau} X^{\alpha^{\prime}}+\mathcal{F}_{\beta^{\prime}}^{\alpha^{\prime}} \partial_{\sigma} X^{\beta^{\prime}}\right)_{\tau_{0}}\left|B^{\prime}\right\rangle=0 \quad, \quad \alpha^{\prime}, \beta^{\prime} \neq \alpha_{c} \\
\left(\partial_{\sigma} X^{\alpha_{c}}\right)_{\tau_{0}}\left|B^{\prime}\right\rangle=0 \\
\left(\partial_{\sigma} X^{i}\right)_{\tau_{0}}\left|B^{\prime}\right\rangle=0
\end{array}\right. \\
& \left\{\begin{array}{l}
\left(\left(\psi^{\alpha^{\prime}}-i \eta \tilde{\psi}^{\alpha^{\prime}}\right)-\mathcal{F}_{\beta^{\prime}}^{\alpha^{\prime}}\left(\psi^{\beta^{\prime}}+i \eta \tilde{\psi}^{\beta^{\prime}}\right)\right)_{\tau_{0}}\left|B^{\prime}\right\rangle=0, \\
\left(\psi^{\alpha_{c}}+i \eta \tilde{\psi}^{\alpha_{c}}\right)_{\tau_{0}}\left|B^{\prime}\right\rangle=0 \\
\left(\psi^{i}+i \eta \tilde{\psi}^{i}\right)_{\tau_{0}}\left|B^{\prime}\right\rangle=0 .
\end{array}\right.
\end{aligned}
$$

We see that these are boundary state equations of a mixed brane with field strength $\mathcal{F}_{\alpha^{\prime} \beta^{\prime}}$ and dimension $p-1$, i.e. $X^{\alpha_{c}}$ is perpendicular to this $\mathrm{m}_{p-1}$-brane. The action of T-duality on one 
of the transverse compact directions of the $\mathrm{m}_{p}$-brane, for example $X^{i_{c}}$, gives a $\mathrm{m}_{p+1}$-brane, i.e. $X^{i_{c}}$ is along this $\mathrm{m}_{p+1}$-brane, which has field strength $\overline{\mathcal{F}}^{\bar{\alpha}}{ }_{\bar{\beta}}$,

$$
\overline{\mathcal{F}}_{\beta}^{\alpha}=\mathcal{F}_{\beta}^{\alpha} \quad, \quad \overline{\mathcal{F}}^{\bar{\alpha}}{ }_{i_{c}}=0
$$

where $\{\bar{\alpha}\}=\left\{i_{c}\right\} \bigcup\{\alpha\}$.

If we simultaneously apply T-duality on two or more directions of a $\mathrm{m}_{p}$-brane, some interesting effects appear. Consider two compact directions of the brane, $X^{\alpha_{0}}$ and $X^{\beta_{0}}$. From the states (15) and (16), we find that the metric and the antisymmetric tensor, under the $\mathrm{T}_{\alpha_{0} \beta_{0}}$-duality transform to

$$
T_{\alpha_{0} \beta_{0}}:\left\{\begin{array}{rl}
G^{\alpha_{0} \beta_{0}} & \rightarrow-G^{\alpha_{0} \beta_{0}}, \\
G^{\alpha_{0} \nu^{\prime}} & \leftrightarrow-B^{\alpha_{0} \nu^{\prime}} \\
B^{\alpha_{0} \beta_{0}} & \rightarrow-B^{\alpha_{0} \beta_{0}}
\end{array}, \quad \nu^{\prime} \neq \alpha_{0}, \beta_{0},\right.
$$

therefore, the boundary state equations (1)-(4) transform to the following forms,

$$
\left\{\begin{array}{l}
\left(\partial_{\tau} X^{\alpha^{\prime}}+\mathcal{F}_{\beta^{\prime}}^{\alpha^{\prime}} \partial_{\sigma} X^{\beta^{\prime}}\right)_{\tau_{0}}\left|B^{\prime \prime}\right\rangle=0 \\
\left(\partial_{\sigma} X^{\alpha_{0}}-\mathcal{F}_{\beta_{0}}^{\alpha_{0}} \partial_{\tau} X^{\beta_{0}}\right)_{\tau_{0}}\left|B^{\prime \prime}\right\rangle=0 \\
\left(\partial_{\sigma} X^{\beta_{0}}-\mathcal{F}^{\beta_{0}} \partial_{0} \partial_{\tau} X^{\alpha_{0}}\right)_{\tau_{0}}\left|B^{\prime \prime}\right\rangle=0 \\
\left(\partial_{\sigma} X^{i}\right)_{\tau_{0}}\left|B^{\prime \prime}\right\rangle=0
\end{array}\right.
$$

for the bosonic part, and

$$
\left\{\begin{array}{l}
\left(\left(\psi^{\alpha^{\prime}}-i \eta \tilde{\psi}^{\alpha^{\prime}}\right)-\mathcal{F}_{\beta^{\prime}}^{\alpha^{\prime}}\left(\psi^{\beta^{\prime}}+i \eta \tilde{\psi}^{\beta^{\prime}}\right)\right)_{\tau_{0}}\left|B^{\prime \prime}\right\rangle=0 \\
\left(\left(\psi^{\alpha_{0}}+i \eta \tilde{\psi}^{\alpha_{0}}\right)+\mathcal{F}_{\beta_{0}}^{\alpha_{0}}\left(\psi^{\beta_{0}}-i \eta \tilde{\psi}^{\beta_{0}}\right)\right)_{\tau_{0}}\left|B^{\prime \prime}\right\rangle=0 \\
\left(\left(\psi^{\beta_{0}}+i \eta \tilde{\psi}^{\beta_{0}}\right)+\mathcal{F}_{\alpha_{0}}^{\beta_{0}}\left(\psi^{\alpha_{0}}-i \eta \tilde{\psi}^{\alpha_{0}}\right)\right)_{\tau_{0}}\left|B^{\prime \prime}\right\rangle=0 \\
\left(\psi^{i}+i \eta \tilde{\psi}^{i}\right)_{\tau_{0}}\left|B^{\prime \prime}\right\rangle=0
\end{array}\right.
$$

for the fermionic part, where $\alpha^{\prime}, \beta^{\prime} \notin\left\{\alpha_{0}, \beta_{0}\right\}$. From these equations we conclude that, if $\mathcal{F}^{\alpha_{0}}{ }_{\beta_{0}}=0$, the directions $X^{\alpha_{0}}$ and $X^{\beta_{0}}$ are perpendicular to the new brane, (therefore it has dimension $p-2)$. In the case $\mathcal{F}_{\beta_{0}}^{\alpha_{0}} \neq 0$, the dimension of the new brane is " $p$ " and field strength on it, is $\mathcal{H}_{\beta}^{\alpha}$, where

$$
\left\{\begin{array}{l}
\mathcal{H}^{\alpha^{\prime}}{ }_{\beta^{\prime}}=\mathcal{F}^{\alpha^{\prime}}{ }_{\beta^{\prime}} \\
\mathcal{H}^{\alpha_{0}}{ }_{\beta_{0}}=-\mathcal{H}^{\beta_{0}}{ }_{\alpha_{0}}=1 / \mathcal{F}_{\beta_{0}}^{\alpha_{0}}, \\
\mathcal{H}^{\alpha^{\prime}}{ }_{\alpha_{0}}=\mathcal{H}^{\alpha^{\prime}}{ }_{\beta_{0}}=0
\end{array}\right.
$$

The field strength $\mathcal{H}^{\alpha}{ }_{\beta}$ is antisymmetric, as expected. Similarly the action of the T-duality on three or more directions of a mixed brane, changes it to another mixed brane. 


\section{$3 \quad \mathbf{m}_{2}$-brane from $\mathbf{D}_{1}$-brane}

Now with appropriate actions of T-duality, we obtain a $\mathrm{m}_{2}$-brane with all the components of $\mathcal{F}$ that are $\mathcal{F}_{01}, \mathcal{F}_{02}$ and $\mathcal{F}_{12}$, from a $\mathrm{D}_{1}$-brane. Consider a $\mathrm{D}_{1}$-brane parallel to the $X^{1}$-direction. The boundary state equations of it, are

$$
\left\{\begin{array}{l}
\left(\partial_{\tau} X^{0}\right)_{\tau_{0}}\left|B_{d}\right\rangle=0 \\
\left(\partial_{\tau} X^{1}\right)_{\tau_{0}}\left|B_{d}\right\rangle=0 \\
\left(\partial_{\sigma} X^{i}\right)_{\tau_{0}}\left|B_{d}\right\rangle=0
\end{array}\right.
$$

for the bosonic part, and

$$
\left\{\begin{array}{c}
\left(\psi^{0}-i \eta \tilde{\psi}^{0}\right)_{\tau_{0}}\left|B_{d}\right\rangle=0 \\
\left(\psi^{1}-i \eta \tilde{\psi}^{1}\right)_{\tau_{0}}\left|B_{d}\right\rangle=0 \\
\left(\psi^{i}+i \eta \tilde{\psi}^{i}\right)_{\tau_{0}}\left|B_{d}\right\rangle=0
\end{array}\right.
$$

for the fermionic part, where $i \in\{2,3, \ldots, 9\}$.

Consider $\bar{X}^{1} \bar{X}^{2}$-plane inside the $X^{1} X^{2}$-plane, which $\bar{X}^{1}$-direction makes angle $\theta$ with the $X^{1}$-direction, therefore

$$
\left\{\begin{array}{l}
X^{1}=\bar{X}^{1} \cos \theta-\bar{X}^{2} \sin \theta \\
X^{2}=\bar{X}^{1} \sin \theta+\bar{X}^{2} \cos \theta \\
X^{0}=\bar{X}^{0} \\
X^{j}=\bar{X}^{j}
\end{array}\right.
$$

where $j \in\{3,4, \ldots, 9\}$. From relations (27) and equations of motion of $\left\{X^{\mu}\right\}$, we see that for $\bar{X}^{\mu}$ there is $\left(\partial_{\tau}^{2}-\partial_{\sigma}^{2}\right) \bar{X}^{\mu}=0$, which gives $\bar{X}^{\mu}=\bar{X}_{L}^{\mu}(\tau+\sigma)+\bar{X}_{R}^{\mu}(\tau-\sigma)$, therefore we can define $\bar{X}^{\prime \mu}=\bar{X}_{L}^{\mu}-\bar{X}_{R}^{\mu}$. Let $X^{1}$ and $X^{2}$-directions be compact on circles of radii $\mathrm{R}_{1}$ and $\mathrm{R}_{2}$. Therefore for appropriate angle $\theta$ we can apply T-duality on $\bar{X}^{1}$ and $\bar{X}^{2}$-directions. Since fermion components $\psi^{\mu}$ and $\tilde{\psi}^{\mu}$ are space-time vectors, behaviors of them under the space-time rotations are like $X^{\mu}$. Therefore, from now on we restrict ourselves to the bosonic part.

Application of relations (27) in equations (25) and then making T-duality on $\bar{X}^{2}$-direction give the following boundary state equations (in the $\left\{X^{\mu}\right\}$-coordinate system),

$$
\left\{\begin{array}{l}
\left(\partial_{\tau} X^{0}\right)_{\tau_{0}}\left|B^{\prime}\right\rangle=0 \\
\left(\cos \theta \partial_{\tau} X^{1}-\sin \theta \partial_{\sigma} X^{2}\right)_{\tau_{0}}\left|B^{\prime}\right\rangle=0 \\
\left(\cos \theta \partial_{\tau} X^{2}+\sin \theta \partial_{\sigma} X^{1}\right)_{\tau_{0}}\left|B^{\prime}\right\rangle=0 \\
\left(\partial_{\sigma} X^{j}\right)_{\tau_{0}}\left|B^{\prime}\right\rangle=0
\end{array}\right.
$$


these equations describe a $\mathrm{m}_{2}$-brane parallel to the $X^{1} X^{2}$-plane, with the field strength $F_{12}=-F_{21}=-\tan \theta$ and $F_{01}=F_{02}=0$.

A $\mathrm{m}_{2}$-brane with non-zero electric and magnetic fields can be obtained from the above $\mathrm{m}_{2^{-}}$ brane by T-duality. A boost $v=\tanh \phi$, along the direction $X^{1}$ gives the $\left\{Y^{\mu}\right\}$-coordinate system,

$$
\left\{\begin{array}{l}
X^{0}=Y^{0} \cosh \phi-Y^{1} \sinh \phi \\
X^{1}=-Y^{0} \sinh \phi+Y^{1} \cosh \phi \\
X^{2}=Y^{2}, \\
X^{j}=Y^{j}, \quad j \neq 0,1,2 .
\end{array}\right.
$$

In this system, the components of field strength are $\mathcal{H}^{\alpha}{ }_{\beta}$, where

$$
\mathcal{H}^{0}{ }_{1}=0 \quad, \quad \mathcal{H}^{0}{ }_{2}=-\sinh \phi \tan \theta \quad, \quad \mathcal{H}^{1}{ }_{2}=-\cosh \phi \tan \theta
$$

therefore

$$
F_{2}^{1}=-\sinh \phi \mathcal{H}_{2}^{0}+\cosh \phi \mathcal{H}_{2}^{1}
$$

Assume that the time direction $X^{0}$ also be compact on circle of radius $R_{0}$. For appropriate values of $\phi$, the coordinates $Y^{0}$ and $Y^{1}$ also are periodic.

Introducing relations (29) and (31) in equations (28), and then making T-duality, simultaneously on $Y^{0}$ and $Y^{2}$ directions (use the transformations (21) to transform field strength $\mathcal{H}$ ), give the boundary state equations of the form (1) and (2) (in the $\left\{X^{\mu}\right\}$-coordinate system) with the field strength,

$$
\left\{\begin{array}{c}
\mathcal{F}^{0}{ }_{1}=\mathcal{F}_{0}{ }_{0}=-\operatorname{coth} \phi \\
\mathcal{F}^{0}{ }_{2}=\mathcal{F}^{2}{ }_{0}=-\frac{\operatorname{coth} \phi}{\tan \theta \sinh ^{2} \phi} \\
\mathcal{F}^{1}{ }_{2}=-\mathcal{F}^{2}{ }_{1}=\frac{1}{\tan \theta \sinh ^{2} \phi}
\end{array}\right.
$$

Note that according to the space-time metric $\eta_{\mu \nu}=\operatorname{diag}(-1,1, \ldots, 1)$, the field strength $\mathcal{F}^{\alpha}{ }_{\beta}$ is antisymmetric.

We obtained a $\mathrm{m}_{2}$-brane which contains non-zero electric and magnetic fields from a $\mathrm{D}_{1}$-brane. More applications of T-duality, on the appropriate directions, lead to the mixed branes with higher dimensions.

Similarly application of T-duality on a direction makes angle with a $\mathrm{D}_{p}$-brane, produces a $\mathrm{m}_{p+1}$-brane with magnetic field, and on some directions (including the time coordinate) of the boosted system, produces electric field on mixed brane. 


\section{Conclusion}

By using the boundary state formalism, we saw that the action of T-duality on one direction of a $\mathrm{m}_{p^{-}}$-brane, changes it to a $\mathrm{m}_{p-1^{-}}$-brane, and on $k$-directions perpendicular to the $\mathrm{m}_{p^{-}}$ brane, changes it to a $\mathrm{m}_{p+k}$-brane, and on some pair of directions of a certain mixed brane, does not change the dimension of it, this action changes the mixed brane to another mixed brane with the modified field strength.

Application of the T-duality on appropriate directions, changes a $\mathrm{D}_{p}$-brane to a mixed brane. By successive applications of the T-duality, we specially obtained a $\mathrm{m}_{2}$-brane from a $\mathrm{D}_{1}$-brane.

\section{Acknowledgement}

The author would like to thank H. Arfaei for useful discussion.

\section{References}

[1] J. Polchinski, S. Chaudhuri, C.V. Johnson, "Notes on D - branes", hep-th/9602052.

[2] R.G. Leigh, Mod. Phys. Lett.A4 (1989)2767.

[3] A. Giveon, M. Porrati, E.Rabinovici, Phys. Rep. 244(1994)77-202.

[4] M. Dine, P. Huet and N. Seiberg, Nucl. Phys.B322(1989)301.

[5] R.R. Khuri, "Spacetime Supersymmetry and Duality in String Theory", hepth/9506086.

[6] L. Thorlacius, Nucl. Phys. Proc. Suppl. 61A(1998)86-98, hep-th/9708078.

[7] J. Dai, R.G. Leigh and J. Polchinski, Mod. Phys. Lett. A4(1989)2073.

[8] H. Arfaei and D. Kamani, Phys. Lett.B452(1999)54, hep-th/9909167.

[9] H. Arfaei and D. Kamani, Nucl. Phys. B561(1999)57-76, hep-th/9911146; Phys. Lett. B475(2000)39, hep-th/9909079.

[10] D. kamani, "Moving Mixed Branes in Compact Spacetime", hep-th/9910043. 